\title{
A Research of Forest Fire Detection Based on the Compressed Video
}

\author{
Weijie Zou ${ }^{1}$, Zijun Guo ${ }^{1}$, Xiaohong $\mathrm{Xu}^{1}$, Yubin Guo ${ }^{1}$, \\ Tonglin Zhu ${ }^{1(\bowtie)}$, and Xinjian Tang ${ }^{2}$ \\ ${ }^{1}$ Institution of Agricultural Multimedia Technology, \\ College of Mathematics and Informatics, South China Agricultural University, \\ Guangzhou, China \\ \{guoyubin, tlzhu\}@scau.edu.cn \\ 2 State Key Laboratory of Geomechanics and Geotechnical Engineering, \\ Institute of Rock and Soil Mechanics, Chinese Academy of Sciences, \\ Wuhan, China
}

\begin{abstract}
The speed of Forest fire detection is important for fire safety. This paper presents a forest fire detection algorithm. At first the images were compressed through combining DCT code and RLE code, then the suspicious images were priority identified at the decoder, at last color identification was detected using HSI color space model flame. Experimental result shows that this algorithm can quickly achieve rapid identification with high accuracy. It has a strong anti-jamming capability and application prospects.
\end{abstract}

Keywords: Forest fires $\cdot$ Fire recognition $\cdot$ Video detection

\section{Introduction}

Fire detection and alarm are important means of fire safety. The traditional fire detection methods used infrared sensor and smoke sensors to detect the parameters such as smoke, temperature and light which were generated during the fire. But those methods have defects such as small space practical, poor anti-interference ability, high cost and respond-limitation. In recent years, video monitoring is widely used in fire detection. Remote computer was used to detect fire which can make up for the inadequacy of traditional method. Video monitoring has the advantage, such as low cost and good practicability, multi-angle, monitoring range and high accuracy, etc.

Domestic and international experts have both made considerable research progress in fire detection. Ye Qingsheng et al. [1] detect the subject area via the difference method using current frame minus reference frame. Razmi S.M. et al. [2], however, detects the suspicious area via picture segmentation using the methods of picture detection and edge detection. Using improved k-mean clustering algorithm, Chakraborty I. et al. [3] recognize the fire image with an accuracy rate as high as $89.8 \%$. However, the algorithm is complex. Tang Yanyan et al. [4] use Gaussian Model with self-adaptive background verification to detect the moving region, and update the model parameters gradually, then use model matching to detect the moving area. Xie Di et al. [5] found that background pixel is distributed normally along time 
axis and started a number of iterations through difference of time domain. However, the algorithm is too time-consuming. Celik T. et al. [6] established a model of fire color based on the statistical analysis towards flame pixels of image foreground information. Zhang Zhengrong et al. [7] segment the color image in HSI space and extract the suspected fire region. However, the false alarm rate is considerably high.

There are a number of research achievements of fire detection algorithm based on video, and it becomes the current hot topic that how to improve the detection efficiency while assuring high detection accuracy. Early detection of fire plays an important role in decreasing economic loss. This article designs a rapid fire-recognition algorithm by adding filter function in the process of video image decoding. Using this method, the key frame of suspected fire can be found more quickly and be decompressed preferentially. Then the areas of significant brightness changes of images will be counted, which will help to position the fire region and realize rapid fire recognition in the end. The experiment verifies this system to be practical and accurate. The experiment result shows that this system possesses a high accuracy and strong anti-interference capability. Therefore, this system has potential application prospects.

\section{HSI Color Model}

HSI model describes the characteristics of the color by Hue, Saturation and Intensity based on human visual system. Compared with the RGB model, the correlation between components of H, S, I in HSI model is small. And it better resembles the visual physiological characteristics of human eyes, for the human eye's ability of distinguishing the changes in HSI is stronger than the changes in RGB. We can see more nature and intuition in HSI space images. Before the fire detection we will transform the image in RGB space into the image in HSI space, which can effectively improve the accuracy of fire detection and recognition speed. Transforming RGB image into HSI image needs a nonlinear transformation, the conversion formula is as follow:

$$
\begin{gathered}
H=\left\{\begin{array}{cc}
\theta, & B \leq G \\
360-\theta, & B>G
\end{array} \quad \theta \in[0,2 \pi]\right. \\
\theta=\arccos \left\{\frac{\frac{1}{2}[(R-G)+(R-B)]}{\left[(R-G)^{2}+(R-B)(G-B)\right]^{1 / 2}}\right\}
\end{gathered}
$$

The saturation component is as follows:

$$
S=1-\frac{3}{(R+G+B)}[\min (R, G, B)]
$$

The intensity component is as follow:

$$
I=\frac{1}{3}(R+G+B) \quad I \in[0,255]
$$


A lot of experiments were carried on to detect fire picture in HSI space by many researchers. Homg W.B. et al. [8] and Celik T. et al. [9] put forward the experience threshold of the forest fire detection in their literature. These thresholds can be roughly divided into two categories based on the color information and brightness of the image (Table 1):

Table 1. The range of the threshold

\begin{tabular}{l|l|l|l}
\hline The range of the threshold & $0<\mathrm{H}<0.17$ & $0.2<\mathrm{S}<1$ & $120<\mathrm{I}<255$ \\
\hline Under bright background & $0-60^{\circ}$ & $0.4-1$ & $127-255$ \\
\hline Under dark background & $0-60^{\circ}$ & $0.2-1$ & $100-255$ \\
\hline
\end{tabular}

\section{Algorithm Design}

Fire recognition algorithm based on video image is pretty mature in the current. Summarizing the research achievements of the predecessors, the fire recognition algorithm follows these steps of distal-end video compression and transport, video image restoration, image pre-processing, image recognition and early warning. This article mainly discusses the two steps of video compression and restoration, which will shorten the processing time, and recognize the suspected image during the decompression preferentially, which can improve the detection efficiency of the whole system in the end.

This Algorithm will divide several continuous pictures into I frame and $\mathrm{P}$ frame when encoding continuous images. The I frame belongs to intra-frame coding, which compresses the intra-frame coding image of data transferred by eliminating redundant information of image space as much as possible. The $\mathrm{P}$ frame is forward predictive-coded frames, which compresses the coding image of data transferred by reducing the time redundant information sufficiently of the forward coded image frame in the image serial. The frame, called residual frame, includes the codes of the different part only, which is produced by I frame before reference. Residual frame, hereinafter referred to as $\mathrm{C}$ frame, is the corresponding image frame of the difference matrix obtained by comparing the corresponding matrixes of two image frames, which is the $C_{n}=I-P_{n}(n=1,2,3,4 \ldots)$. Transferring $\mathrm{C}$ frame among the image frame will improve compression efficiency.

The algorithm process of this article is as below (Fig. 1):

Step 1. Compress the frames which have been divided from video. This method ensures the independence of picture information, which helps the analysis of picture information. Mark the first frame as I frame. The corresponding $\mathrm{C}$ frame can be obtained by using the other $\mathrm{P}$ frame minus $\mathrm{I}$ frame. Figure 2 is partial $\mathrm{C}$ frame schematic diagram, the first picture of which is I frame. It can be seen from Fig. 2 that the flame shape is clearly displayed in $\mathrm{C}$ frame. The $\mathrm{C}$ frame can detect the fire of image and remove the background.

Step 2. Compress and encode the image. The image quality of I frame will influence the image restoration of other frames. Therefore, we cannot take the usual practice of 


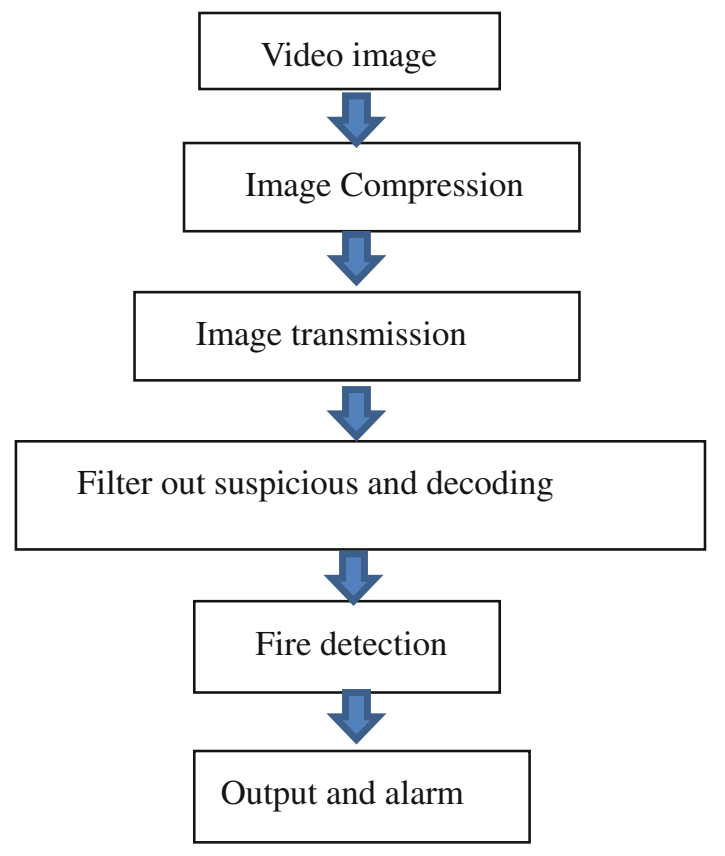

Fig. 1. Algorithm process of fire detection
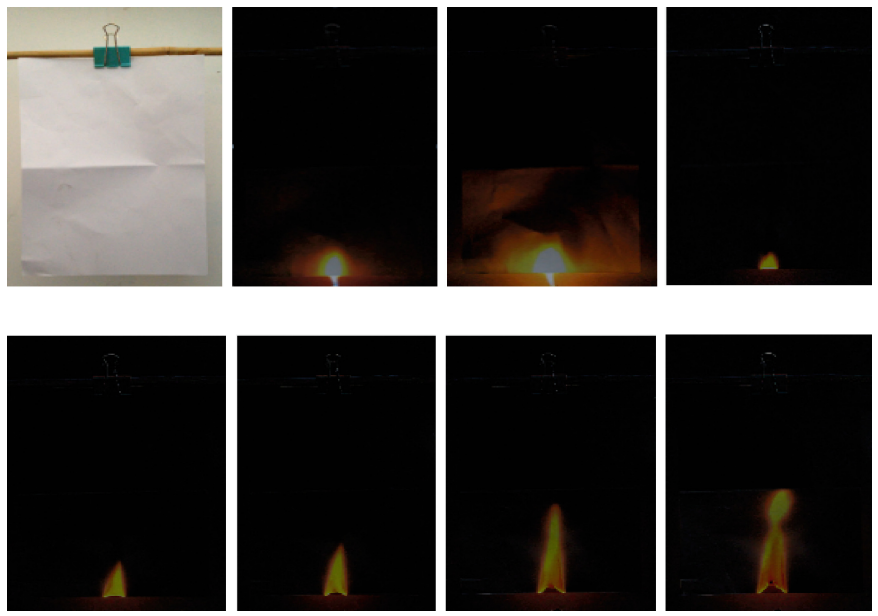

Fig. 2. Residual video frame of simulative fire experiment in the lab

JPEG method. This article chooses the no-damage compression method of DCT and RLE mixed coding, which is easy and efficient. It will reduce the complexity of calculation in the decoding end by using the same compression coding to compress the $\mathrm{C}$ frame. It doesn't require loading other decoding rules from the decoding end to 
decode, which improves the decoding efficiency. In the other hand, after the conversion of DCT, the matrix obtained from residual frame will come out a number of zero parameters. RLE encoding method has very high compression ratio for repeated data.

Step 3. The bit will be transmitted after the encoding. As this experiment is practiced in campus network with unstable network transmission, we set the transmission value as 10 frames each time. The number of frames transferred each time could be increased in actual practice.

Step 4. Decodes suspected fire frame. Firstly, find out the longest frame code, then decode RLE. For the RLE codes of residual image, if residual image contain more information, the code length of the codes will be longer. Then make inverse-DCT transformation towards decoding frames. At last determine whether the frame is suspected frame according to the DCT coefficient of image based on the threshold value.

Figure 3 DCT coefficient histogram of C frame is the DCT coefficient histogram of C frame. The left figure is the DCT coefficient histogram of the residual between I frame and P frame without fire. It is clear that its DCT coefficient mostly concentrate around 0 , and no more than 0.5. The right figure is the DCT coefficient histogram of the residual between $\mathrm{P}$ frame and I frame after the fire. Compared with the left picture, the DCT coefficient shows bigger fluctuation, many coefficient value are more than 0.5 . Therefore, we set the threshold value as 0.5 in this experiment. For a residual image with size of $1024 * 1024$, if there are more than 64 values with DCT coefficient more than 0.5 , we consider it as a fire image, which needs to be further recognized.
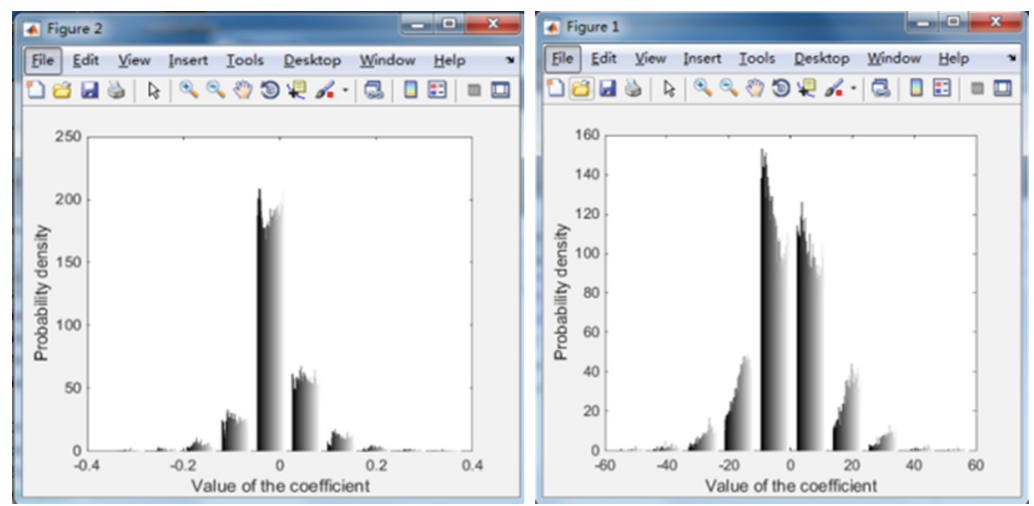

Fig. 3. DCT coefficient histogram of residual video frame

At last, the residual image has been transformed from RGB space to HSI space, and has been divided to several blocks. Suppose the residual image is divided into 320 blocks. We count the pixels with the intensity component more than 80 among the sub-blocks and record its number as $Q_{n}$, in which n represents the code of sub-block. Rank the sub-blocks on the basis of $Q$ value in a descending order. After this, we restore the image of those sub-blocks with larger $\mathrm{Q}$ value and fill color for other 
sub-blocks as grey. Restore 60 sub-blocks of image, and the result was shown in Fig. 4, so the suspected fire region was positioned in this step. However, as the light was produced in the experiment, the flames appear reflect on the metal clip used for fixing, so wrong image blocks are restored above the flames, which is interfered the experiment results. No metal objects of this type will appear in practical inspection environment. However, if it does exist, then we shall eliminate the interference of objects which can reflect things like metal or other objects, and then position the fire region correctly (Fig. 5).
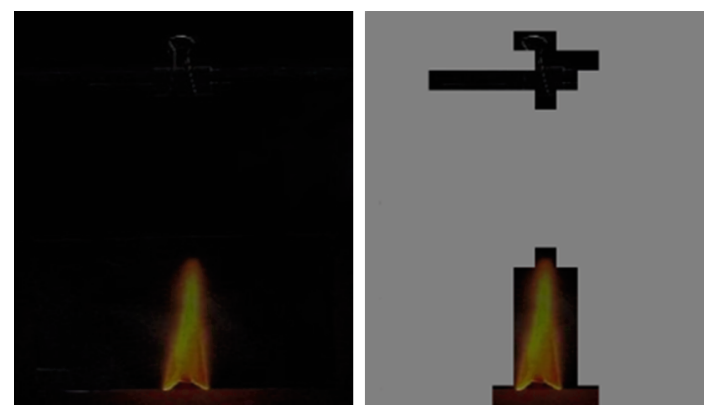

Fig. 4. Suspected flame area of experimental video

\begin{tabular}{llllllll}
\hline $\begin{array}{l}\text { Block } \\
\text { number }\end{array}$ & weight & $\begin{array}{l}\text { Block } \\
\text { number }\end{array}$ & weight & $\begin{array}{l}\text { Block } \\
\text { number }\end{array}$ & weight & $\begin{array}{l}\text { Block } \\
\text { number }\end{array}$ & weight \\
\hline 1 & 20 & 21 & 1 & 41 & 6 & 61 & 0 \\
2 & 22 & 0 & 42 & 2 & 62 & 2 \\
3 & 0 & 23 & 0 & 43 & 0 & 63 & 0 \\
4 & 5 & 24 & 13 & 44 & 0 & 64 & 0 \\
5 & 0 & 25 & 8 & 45 & 0 & 65 & 20 \\
6 & 0 & 26 & 0 & 46 & 0 & 66 & 0 \\
7 & 0 & 27 & 0 & 47 & 0 & 67 & 0 \\
8 & 2 & 28 & 4 & 48 & 0 & 68 & 0 \\
9 & 8 & 29 & 0 & 49 & 20 & 69 & 3 \\
10 & 0 & 30 & 0 & 50 & 0 & 70 & 0 \\
11 & 0 & 31 & 0 & 51 & 0 & 71 & 0 \\
12 & 0 & 32 & 0 & 52 & 0 & 72 & 0 \\
13 & 0 & 33 & 20 & 53 & 0 & 73 & 1 \\
14 & 0 & 34 & 0 & 54 & 0 & 74 & 0 \\
15 & 0 & 35 & 0 & 55 & 0 & 75 & 2 \\
16 & 0 & 36 & 0 & 56 & 0 & 76 & 0 \\
17 & 20 & 37 & 5 & 57 & 2 & 77 & 0 \\
18 & 0 & 38 & 0 & 58 & 1 & 78 & 0 \\
19 & 0 & 39 & 0 & 59 & 0 & 79 & 5 \\
20 & 0 & 40 & 0 & 60 & 0 & 80 & 0 \\
\hline
\end{tabular}

Fig. 5. The statistical table of fire image brightness changes (Part of the data)

At last, color threshold method was used to recognize the suspected region and determine whether a fire happens. The alarm will ring if a fire happens. 


\section{Experiment Result and Analysis}

A few typical fire video and pictures were used to test the forest fire recognition algorithm based on video compression in this article. Most videos for experiment are recorded in laboratory, and the others are from UltimateChase.com. The pictures used in the experiment are from the internet (http://signal.ee.bilkent.edu.tr/VisiFire/). The configuration of computers in laboratory is the Pentium 4 processor. The internal storage is 4G. Operating system is Windows 7. Program applied is the mixing of matlab2014b and Visual $\mathrm{C}++$ 6.0. The version of OpenCV is 1.0.

To prove that the algorithm in this article could recognize fire in all-weather surveillance video, we have designed an experiment of burning papers under different brightness and simulate the scenarios of fires in different periods of time. As shown in Fig. 6, the pictures in the first row are the screenshots taken in normal conditions. In the second row are the suspected frames which were found in detection, and the time of video recording was showed in the bottom. Those fire videos have been successfully determined in our experiment. The experiment shows that the change of background light source has little influence on the fire recognition in this algorithm.
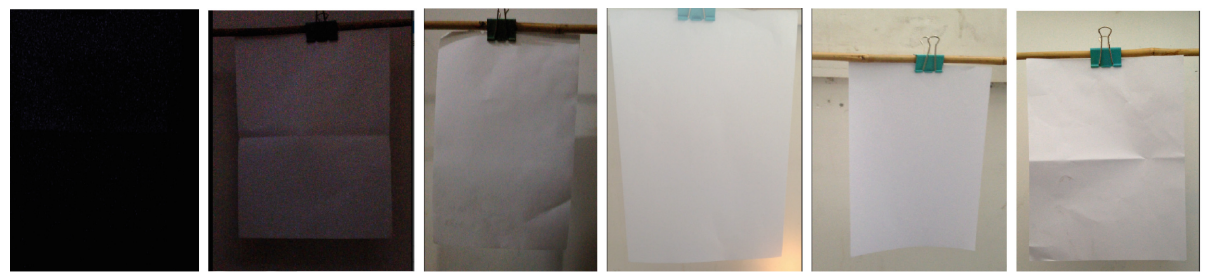

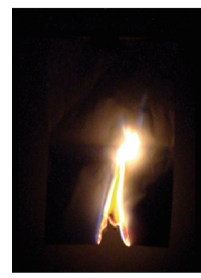

20:00

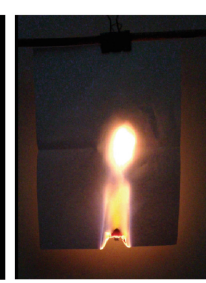

18:00

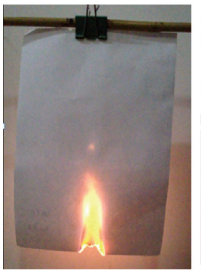

16:00

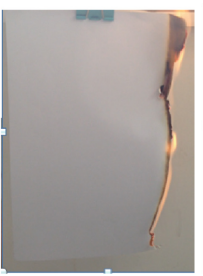

14:0 0

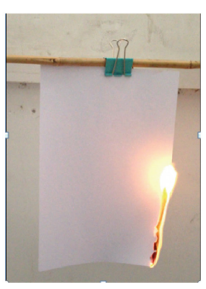

12:00

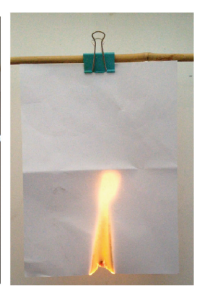

10:00

Fig. 6. Burning experiment screenshots under different brightness

Figure 7 is the image come from four typical fire video. (a) is indoor fire experiment video; (b) is outdoor dead leaves ignition video; (c) is field forest fire video of long distance; (d) is field forest video of short distance. Furthermore, comparative tests were made in this article. Almost all fire recognition algorithm and decoding progress are separated. Recognition after image decoding was recorded as method 1, and recognition during the decoding, applying artificial neural network method from literature [10] was recorded as Method 2. Detailed data of experiment was analyzed and shown as below (Table 2): 


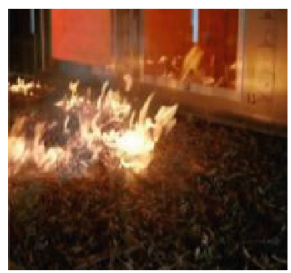

(a)

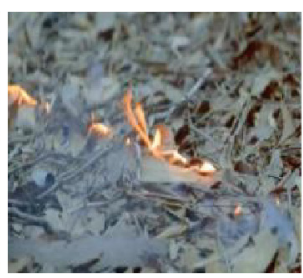

(b)

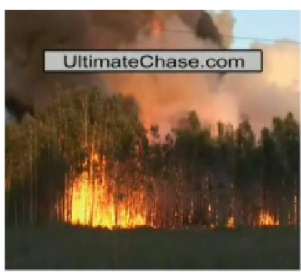

(c)

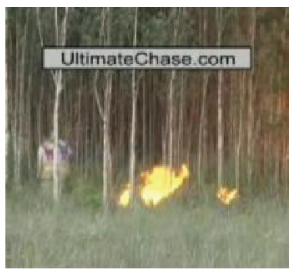

(d)

Fig. 7. Images come from four typical fire videos

Table 2. Comparison of experiment results

\begin{tabular}{l|l|l|l|l|l|c}
\hline Video & Frame & \multicolumn{3}{|l|}{ Fire detection time (s) } & \multicolumn{2}{l}{ Save time ratio } \\
\cline { 3 - 7 } & $\begin{array}{l}\text { rate } \\
\text { (f/s) }\end{array}$ & Method 1 & Method 2 & $\begin{array}{l}\text { Proposed } \\
\text { method }\end{array}$ & $\begin{array}{l}\text { Compared to } \\
\text { the method 1 }\end{array}$ & $\begin{array}{l}\text { Compared to } \\
\text { the method 2 }\end{array}$ \\
\hline video1 & 10 & 3.76 & 3.21 & 3.05 & $18.88 \%$ & $5.00 \%$ \\
\hline video2 & 10 & 3.53 & 3.26 & 2.95 & $16.43 \%$ & $9.51 \%$ \\
\hline video3 & 15 & 4.15 & 3.55 & 3.27 & $21.20 \%$ & $7.89 \%$ \\
\hline video4 & 20 & 4.63 & 3.89 & 3.12 & $32.61 \%$ & $19.79 \%$ \\
\hline
\end{tabular}

From the experiment result, we can see the recognition time of fire in this method is much shorter than the time in the other two methods. So it is a method of fast fire detection. We can use the pre-warning time difference between the two algorithms to divide the minuend to compute the saving time ratio. Compared with the method 1, our method is better in detection efficiency. The saved time in our method is from $0.58 \mathrm{~s}$ to $1.51 \mathrm{~s}$, and the corresponding saving time ratio is from $16.43 \%$ to $32.61 \%$. The quicker transferring frame frequency, the more time saved. The main reason is that the frame frequency transported is related with decoding time, which means the decoding time will be longer with a quicker frame frequency. Under the wideband environment of $100 \mathrm{M}$, the transferring frame frequency can be up to more than 20f/s. All of this can reflect the advantages of rapid recognition of this system. Compared with Method 2, the pre-warning time of these two methods is pretty close. However, our method still has a winning of $0.16 \mathrm{~s}$, with $5 \%$ saving time ratio. The reason is that our method is less complex and more quickly in recognition. The method adopted in this article can detect the flame and alarm within $3.5 \mathrm{~s}$ since the fire started, so it can be used in fire pre-warning in surveillance video.

To prove the accuracy of the fire recognition algorithm in the paper, we collect 200 experiment pictures on the internet to test the algorithm. Figure 8 displays partial image including some representative interference image as sunset, sunset clouds, and street lights etc. The experiment result shows the accuracy is as high as $86 \%$, in which 172 pictures have correct detection result, and 28 pictures have wrong detection result, and 0 pictures have false negative detection result. Besides, this algorithm is also capable of recognizing normal interference image and displaying as no fire. The experiment shows that the system has a considerably high accuracy of fire images, up to more than $86 \%$. 

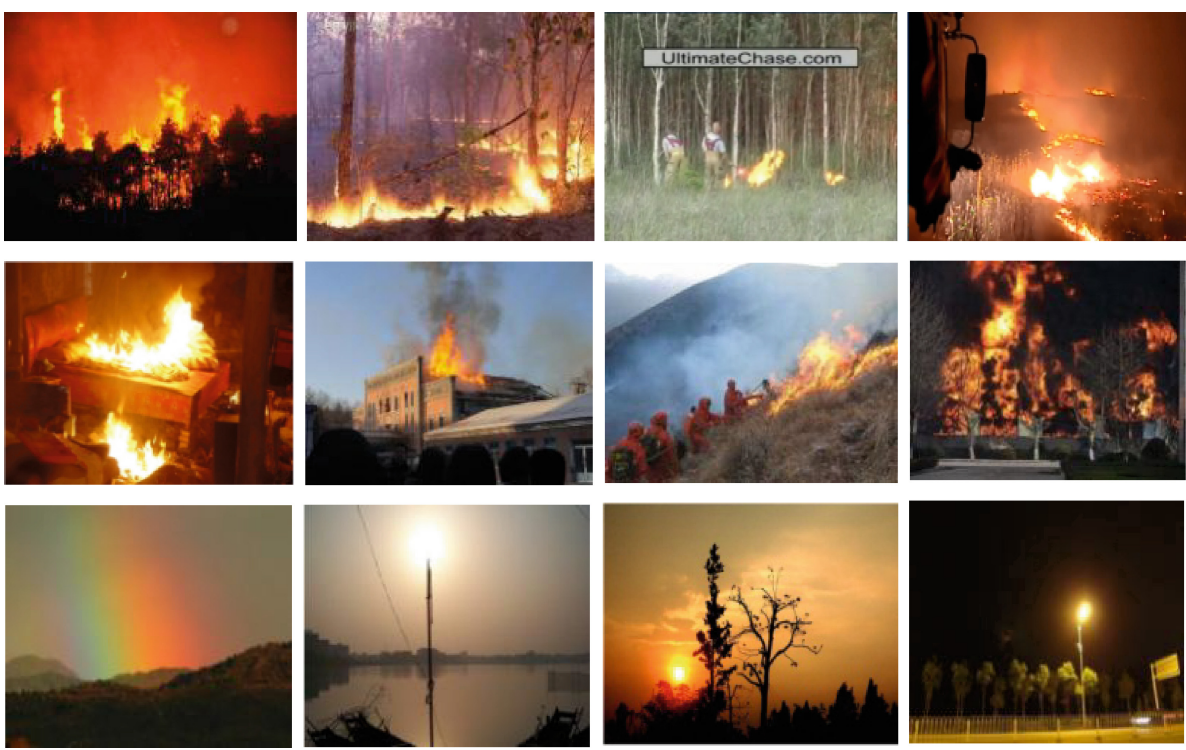

Fig. 8. Experimental image come from internet http://www.chinadaily.com.cn

\section{Conclusion and Future Work}

A rapid recognition method of fire image based on image compression specific was put foreword to solve the dilemma of accuracy and rapidness of current fire detection algorithm. Filter function was set in the unpacking end to filter the code stream which has been delivered in place. The most suspected fire image was unpacked, and then fire detection was recognized by the color threshold method. This method changes the traditional method of unpacking and recognizing frames one by one, reduces the recognizing time, and improves program efficiency. The experiment result on public test videos shows that this method can achieve rapid recognition and pre-warning with high accuracy, which promises a bright application prospect. The recognition method is reliable, small computational amount, fast operation and meets the need of real-time fire judging.

However, the method in this article still requires unpacking at least one frame of image to recognize. The future development direction is to recognize directly under code stream without unpacking at all. We will determine the circularity, sharp corners, growth of area and the center of mass movement as the characteristics extraction object within the detection accuracy. More early fire smoke detection experiments will be done to improve the accuracy and real-time of image-type fire detection.

Acknowledgments. This work was supported by the Supported by Science and Technology Planning Project of Guangdong Province, China Research on Terrain monitoring technology on subway surrounding buildings (2013B010401020), National Natural Science Foundation of China (Grant No. 41372317), The State Forestry Administration of forestry (LY-2013-146). The authors would like to thank the associate editor and reviewers for their comments which are very helpful in improving the revision. 


\section{References}

1. Ye, Q.S., Wang, W.H., Liu, S.Q.: Design and implementation of fire detection with video. J. Comput. Eng. Des. 775-778 (2008)

2. Razmi, S.M., Saad, N., Asirvadam, V.S.: Vision-based flame analysis using motion and edge detection. In: Intelligent and Advanced Systems, Malaysia (2010)

3. Chakraborty, I., Paul, T.K.: A hybrid clustering algorithm for fire detection in video and analysis with color based thresholding method, Bangalore, Karnataka, India (2010)

4. Tang, Y., Yan, Y.Y., Liu, Y.A.: Fast flame detection with GMM. J. Comput. Sci. 283-285 (2012)

5. Xie, D., Tong, R.F., Tang, M.: Distinguishable method for video fire detection. J. Zhejiang Univ. (Eng. Sci.) 46, 698-704 (2012)

6. Celik, T., Demirel, H., Ozkaramanli, H.: Fire detection in video sequences using statistical color model. J. Vis. Commun. Image Represent. 18, 176-185 (2006)

7. Zhang, Z.R., Li, G.G.: Fire detection technology based on support vector machine. J. Microcomput. Appl. 29, 70-72 (2010)

8. Horng, W.B., Peng, J.W., Chen, C.Y.: A new image-based real-time flame detection method using color analysis. In: Networking, Sensing and Control, pp. 100-105 (2005)

9. Celik, T., Ma, K.K.: Computer vision based fire detection in color images. In: Soft Computing in Industrial Applications, Muroran, pp. 258-263 (2008)

10. Golomb, S.J.: Run-length encodings. IEEE Trans. Inf. Theor. 12, 399-401 (1966) 